\title{
Regulation of autophagy and EMT by the interplay between p53 and RAS during cancer progression (Review)
}

\author{
XIAOFEI ZHANG ${ }^{1,2^{*}}$, QIAN CHENG ${ }^{3 *}$, HUIJING YIN ${ }^{1,2}$ and GONG YANG ${ }^{1,2,4}$ \\ ${ }^{1}$ Cancer Institute, Fudan University Shanghai Cancer Center; \\ ${ }^{2}$ Department of Oncology, Shanghai Medical College, Fudan University, Shanghai 200032; \\ ${ }^{3}$ Department of Orthopedics, The Affiliated Hospital of Jiangsu University, Zhenjiang, Jiangsu 212001; \\ ${ }^{4}$ Central Laboratory, The Fifth People's Hospital of Shanghai Fudan University, Shanghai 200240, P.R. China
}

Received February 23, 2017; Accepted May 5, 2017

DOI: $10.3892 /$ ijo.2017.4025

\begin{abstract}
Cellular autophagy and epithelial-mesenchymal transition (EMT) are key events mostly resulted from the interplay of tumor suppressors and oncogenes during cancer progression. The master tumor suppressor p53 may control tumor cell autophagy and EMT through the transcriptional induction of multiple target genes, while the activated oncogene RAS may also play a critical role in regulating mitogenic signaling to tumor cell autophagy and EMT. Although the fundamental functions of p53 and RAS are well understood, the interactive effects of p53 and RAS on autophagy and EMT are still unclear. In this review, we highlight the recent advances in the regulation of autophagy and EMT by p53 and RAS, aiming to explore novel therapeutic targets and biomarkers in cancer treatment and prevention.
\end{abstract}

\section{Contents}

1. Introduction

2. Mutations of p53 and RAS synergistically promote cell autophagy

3. p53 and RAS participate in regulation of cancer cell EMT

4. The relationship between autophagy and EMT in cancer

5. Perspectives

\section{Introduction}

In response to various stresses such as DNA damage or hypoxia, the tumor suppressor p53 can be activated to

Correspondence to: Professor Gong Yang, Cancer Institute, Fudan University Shanghai Cancer Center, 270 Dong'an Road, Shanghai 200032, P.R. China

E-mail: yanggong@fudan.edu.cn

*Contributed equally

Key words: p53, RAS, autophagy, epithelial-mesenchymal transition regulate cell cycle, differentiation, apoptosis, senescence and autophagy $(1,2)$. Mutations of p53 in single allele may lead to loss of the tumor suppressor functions, gain of oncogenic functions, or exert dominant-negative effects which may disrupt the normal functions of the wild-type allelic p53 (3). Under normal circumstances, p53 is rapidly turned over by ubiquitinization through binding to MDM2. Mutant p53 is usually much more stable than the wild-type p53 due to the loss of the binding activity to MDM2, and is often accumulated in tumor cells $(4,5)$. While the wild-type p53 predominantly functions as a transcription factor, the mutant p53 also has the ability to transactivate multiple genes involved in cell proliferation, apoptosis inhibition, chemoresistance and matrix degradation (4).

On the other hand, oncogenic mutations of RAS are detected in many cancer types including pancreatic, lung, ovarian and colon cancers, which usually lead to chemo- and/ or radio-resistance of cancer cells $(6,7)$. RAS activates several downstream cascade branches including the RAF/MEK/ ERK, PI3K/AKT and RalGDS/Ral signal molecules critical for cancer progression $(8,9)$. Although p53 and RAS are individually reported to contribute to cellular autophagy and EMT, how they functionally interact with each other to cooperatively regulate the downstream signaling cancer progression is unclear. In this mini review, we will summarize the recent findings regarding the functional interaction of mutant p53 and RAS in modulating cancer progression through some key events of cell autophagy and EMT (10-13).

\section{Mutations of p53 and RAS synergistically promote cell autophagy}

Autophagy, an intracellular catabolic process in response to stress and nutrient deprivation, plays multiple roles during tumorigenesis and cancer therapy (14). To maintain metabolic homeostasis, autophagy occurs to deliver excessive or unnecessary cytoplasmic components as well as injured or aged organelles to the lysosomes for degradation $(15,16)$. As a homeostatic process, autophagy has both tumor-promoting and tumor-suppressing properties depending on cancer cell type and the tumorigenic context (17). The main regulators of autophagy include the PI3K-Akt-mTOR pathway associated 
molecules, RAS and p53 (14). Several studies have shown that the nuclear p53 stimulates cellular autophagy via the transactivation of multiple target genes, while the cytoplasmic p53 inhibits autophagy in a transcription-independent manner, therefore the subcellular localization of p53 may determine the outcome of autophagy $(18,19)$. On the other hand, RAS can modulate autophagy via various signaling cascades in cancer cells, conversely, autophagy also mediates and promotes the RAS-driven cancer progression and invasion $(20,21)$. RAS renders mitochondrial health particularly reliance on autophagy to the extent that RAS-driven cancer cells seem more autophagy-dependent for survival to nutrient starvation than normal cells. Thus, that RAS-driven cancers are susceptible to autophagy inhibition therapy (22).

Both Ras and p53 are reported to interact with several identical binding partners and signaling cascades during the autophagy process, suggesting the possible interplay between their corresponding pathways. In the nucleus, p53 activates Sestrin1 (also known as p53-activated gene 26, PA26) and Sestrin2 (also known as hypoxia-inducible gene 95, Hi95), to induce autophagy through the activation of adenosine monophosphate-activated protein kinase (AMPK) (23). The activated AMPK inhibits mTOR1 activity by phosphorylating the mTORC1 binding factor Raptor or the tumor suppressor tuberous sclerosis protein $1 / 2$ (TSC1/2) complex $(24,25)$. Studies on metastatic pancreatic ductal adenocarcinomas showed that two K-RAS activation pathways RAF/MEK/ERK and PI3K/AKT also converge to the TSC1/2 (26). In addition, inhibition of mTORC1 or activation of AMPK can activate the unc-51-like autophagy activating kinase $1 / 2$ (ULK1/2) to eventually initiate autophagy $(27,28)$.

The autophagy related genes (ATG) have been recognized to execute autophagy directly and the ATG proteins play pivotal roles in the formation of the autophagosomes (29). p53 and RAS regulate autophagy mostly relying on these ATG proteins. In vitro studies on various cancer cell lines showed that overexpression of the p53 target gene Isg20L1 promotes autophagy that can be partially rescued by ATG5 depletion (30). The nucleus p53 induces autophagy through direct activation of serial genes such as ULK1, ULK2 and ATG7 in multiple cell lines such as MEFs, lung cancer cells and HCT116 cells $(31,32)$, indicating that the nucleus p53 induces autophagy at least partially relying on ATG5/7. A recent study on ATG7-deletion genetically engineered mouse models of K-RAS ${ }^{\mathrm{G} 12 \mathrm{D}}$-driven on small-cell lung cancer (NSCLC) showed that the functional status of p53 determined the metabolic requirement for autophagy. During tumor development, intact p53 with ATG7 deletion leads to the premature p53 induction and blocks tumor proliferation, while p53 loss of function restored the proliferation and growth during ATG7 deletion (33). Finally, both H-RAS ${ }^{\mathrm{V} 12}$ and K-RAS ${ }^{\mathrm{V} 12}$ can initiate autophagy by upregulating ATG5 and ATG7 through the Rac1/ mitogen-activated kinase kinase 7 (MKK7)/c-Jun N-terminal kinase (JNK) signaling pathways in normal fibroblasts and human breast epithelial cell line MCF10A (34,35).

Activated RAS and mutant p53 may synergistically regulate autophagy. In human pancreatic cancer cell lines CAPAN-2, PANC-1 and Panc10.05, activated K-RAS and p53 loss of function collaboratively upregulate Plac8 to facilitate autophagosome-lysosome fusion (36). Both RAS and p53 signaling pathways can regulate the heat shock transcription factor 1 (HSF1) that stimulates autophagy through direct binding to the ATG7 promoter and activating its expression during breast cancer progression $(37,38)$. The RAS/RAF/ MEK/ERK signaling pathway activates HSF1 through its phosphorylation at Ser326 in human neurofibrosarcoma cell line MpNST while HSF1 and p53 interfere with each other during cancer development (39). The HSF1 signaling usually depends on p53 mutation status and HSF1 is also required for the nuclear localization of p53 in multiple cell lines $(38,40)$.

Studies have also shown that autophagy plays a cardinal role in response to hypoxia microenvironment of tumors. For example, the hypoxia-inducible factor- $1 \alpha$ (HIF-1 $\alpha$ ) can activate autophagy and alter cancer metabolism (41). During anti-angiogenic therapy, some cancer cells activated both AMPK and HIF-1 $\alpha$ pathways to initiate autophagy and thus survive under the hypoxic insult (42). A study revealed that H-RAS can transform Rat1 fibroblasts through upregulation of HIF-1 $\alpha$ expression, but treatment with either MAPK or PI3K inhibitors suppresses the HIF-1 $\alpha$ level (43). HIF-1 $\alpha$ also stabilizes p53 through direct interaction with and inhibition of MDM2 $(44,45)$. Although it is known that HIF-1 $\alpha$ interacts with both RAS and p53 in hypoxia conditions, how these interactions induce autophagy is still unclear. The detailed signaling pathways involving p53 and RAS in autophagy are presented in Fig. 1.

\section{3. p53 and RAS participate in regulation of cancer cell EMT}

Epithelial-mesenchymal transition (EMT) is a process of certain cells switching from an epithelial to a mesenchymal status (46). During EMT, epithelial cells lose their characteristics as apical-basal polarity and tight junction but gain the mesenchymal properties such as reduced intercellular adhesion and increased motility (47). EMT may play an important role in the initiation and development of cancers and chemoresistance of metastatic cancers (47-50).

It is well established that oncogenic RAS promotes EMT in collaboration with other pathways including p53 (51,52). p53 inhibits the RAS-mediated EMT and EMT-associated stemness of human mammary epithelial cells via the RAS/RAF/ MEK/ERK and the RAS/PI3K/AKT pathways. Moreover, inhibition of the RAS/RAF/MEK/ERK pathway upregulates E-cadherin and $\beta$-catenin expression (53). Both RAS/PI3K/ AKT and RAS/RAF/MEK/ERK pathways stimulate EMT through the activation of Snail2 (also known as Slug) expression and the reduction of E-cadherin in multiple cell lines including colorectal carcinoma cells HCT-116, HKe-3 and $\mathrm{HKh}-2$, rat parotid gland epithelial cell $\mathrm{Pa} 4$, and endometrial cancer cell lines Ishikawa and Hec251 (51,54,55). In nonsmall cell lung cancer, mutation of p53 is associated with high expression of Slug and low expression of E-cadherin, leading to poor prognosis of patients (56). The study suggested that wt p53 can bind to MDM2 and Slug simultaneously to form a p53-MDM2-Slug complex, which then facilitates MDM2mediated Slug degradation (56). The H-RAS ${ }^{\mathrm{V} 12}$-induced EMT can be inhibited by ASPP2 without p53 binding (57). In mouse primary kidney epithelial cells, ASPP2 represses ZEB1 expression by forming ASPP2- $\beta$-catenin-E-cadherin ternary 


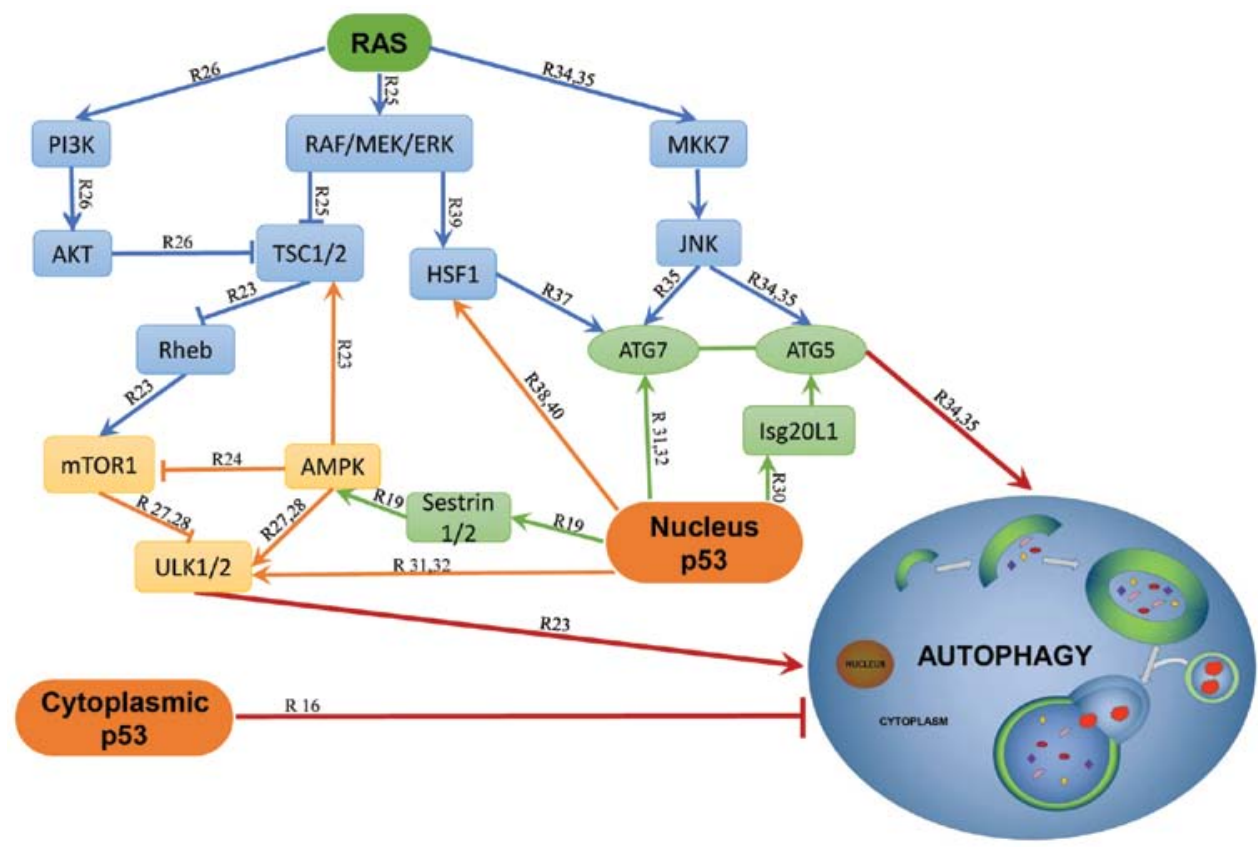

Figure 1. Mutant p53 and RAS synergistically promote cell autophagy. R, reference

complex at cell-cell junctions to negatively regulate the WNT signaling (57). Although ASPP2 suppresses ZEB1 without regard to the p53 mutation status, in hepatocellular carcinoma cell lines and immortal normal mammary epithelial cells, p53 represses ZEB1 and ZEB2 expression through the transcriptional activation of the miRNA-200 family members $(58,59)$. In murine and human cancer cells, Twist 1 and Twist 2 may also cooperate with $\mathrm{H}-\mathrm{RAS}^{\mathrm{V} 12}$ to overcome premature senescence of mouse embryonic fibroblasts through inhibition of the p53 pathway and promotion of EMT by suppressing E-cadherin and stimulating vimentin expression (60).

Concurrent mutations of RAS and p53 have been found to play a critical role in EMT and tumor metastasis via multiple pathways. The Raf kinase trapping to Golgi (RKTG), the negative regulator of the RAS/RAF/MEK/ERK pathway, may also collaborate with p53 to regulate EMT (61). Concomitant knockdown of p53 and RKTG in mice contribute to skin cancer development and epidermal EMT. Studies of A431 and HepG2 cells suggested that loss of p53 and PKTG at the same time reduced E-cadherin but increased vimentin to promote EMT (61). Furthermore, the AKT activator IGF-1 induces EMT with p53 silencing while the AKT inhibitor VIII blocks the E-cadherin $/ \beta$-catenin complex formation induced by 553 and RKTG, implicating that the RAS/PI3K/AKT cascades enhance EMT function likely through inhibition of the p53 function (61). On the other hand, miR-200 blocks EMT and metastasis in syngeneic mice with metastatic lung adenocarcinoma carrying both K-RAS ${ }^{\mathrm{G} 12 \mathrm{D}}$ and $\mathrm{p} 53 \mathrm{R} 172 \mathrm{H} \Delta \mathrm{G}$ mutations (62). Several studies have shown that loss of p53 can enhance the RAS signaling induced EMT. p53 may act as a checkpoint controller to inhibit EMT while loss of p53 allows other signal cascades such as RAS activation to induce EMT (61,63-65). Activation of K-RAS ${ }^{\mathrm{V} 12}$ and loss of p53 may cooperate to induce EMT and cell motility by triggering the RhoA activity (10). In metastatic mouse models, depletion of the Rho-GTPase Rnd1 inhibits the RAS/RAF/MEK/ERK pathway to promote EMT in collaboration with the loss of p53 (66).

Hypoxia-induced EMT, in particularly, is well-known in several cancers such as breast, ovarian, hepatocellular carcinomas and oesophageal squamous cell cancer (67-70). HIF-1 $\alpha$ targets several EMT transcriptional factors including Snail, Slug, Twist and ZEB in hypoxia conditions (71). In response to hypoxia stress, HIF-1 $\alpha$ can activate PI3K/AKT to promote EMT and to enhance the tumor cell metastatic potential (67). As mentioned above, p53 and RAS may have an intimate crosstalk with HIF-1 $\alpha$, indicating the interactive potentials among the three molecules during EMT or MET.

Since the first step of tumor metastasis is characterized by the increased motility and invasiveness, it has been implicated that EMT plays a cordial role in promoting metastasis, although the role of EMT for invasion and metastasis remains contested (72). Mutant p53 and oncogenic RAS promote EMT while the upregulation of wt p53 suppresses RAS-induced EMT phenotypes. p53 may interact with the RAS signaling to inhibit or promote EMT process via multiple pathways depending on the p53 status and RAS activation level. The detailed signaling pathways involving p53 and RAS in EMT are depicted in Fig. 2.

\section{The relationship between autophagy and EMT in cancer}

Autophagy and EMT are two key processes during cancer progression and linked in a close relationship with each other according to recent studies. The interactions between autophagy and EMT is complicated. Just like its dual role in cancer, autophagy also has two-tier functions on EMT according to the cellular type and the stage of tumor progression (73). Several studies showed the controversial effect of autophagy on EMT. Autophagy inhibition promotes EMT while autophagy activation reverses EMT mainly by regulating several mesenchymal markers. A recent study on gastric cancer cells 


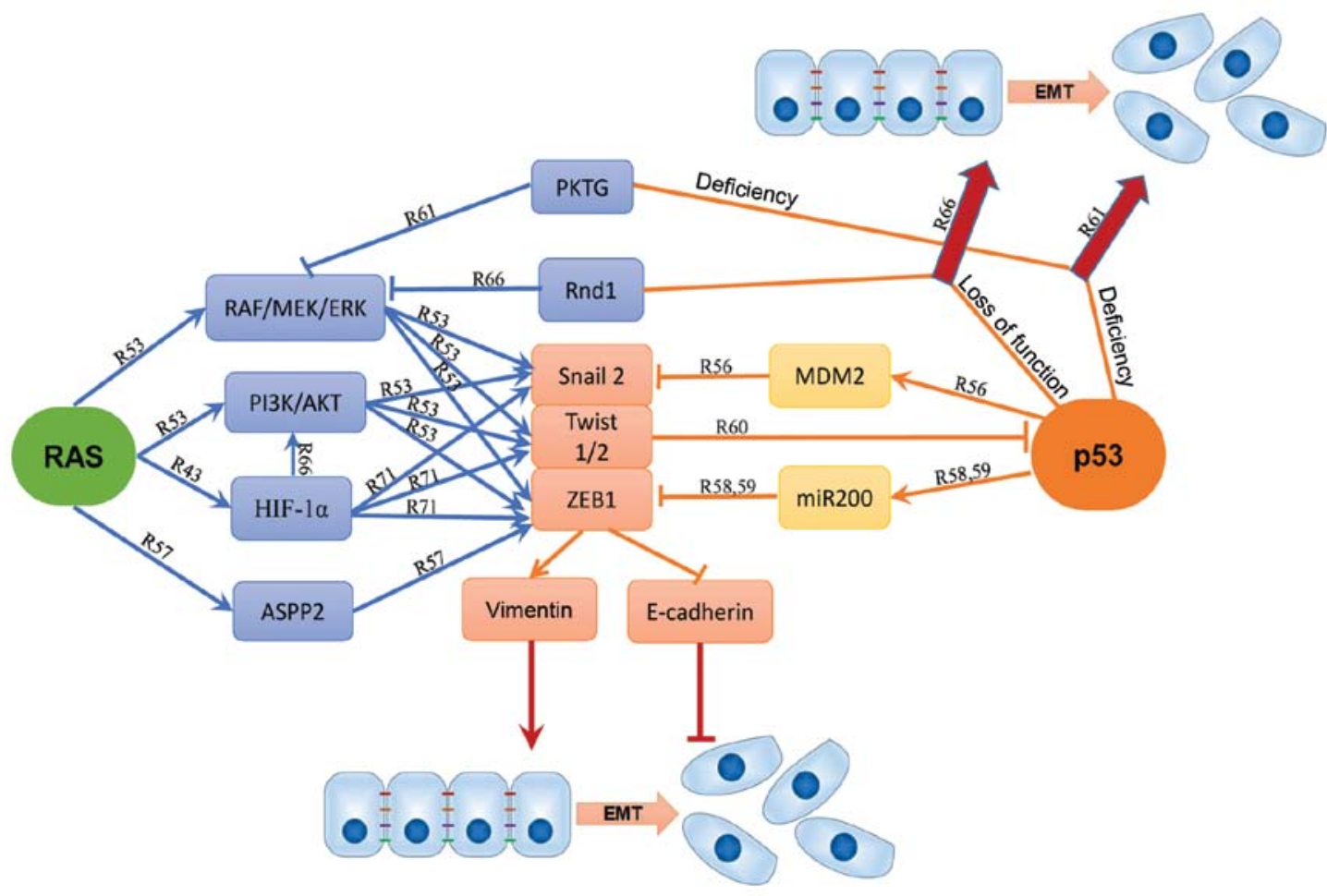

Figure 2. p53 and RAS participate in regulation of cancer cell EMT. R, reference.

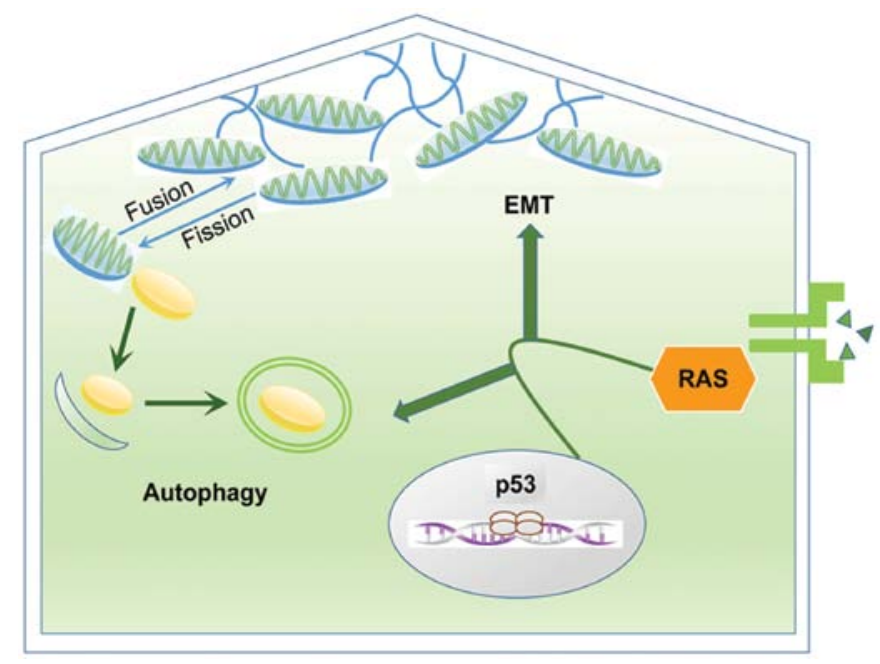

Figure 3. The relationship between autophagy and EMT in cancer.

indicated that autophagy deficiency increases the expression of mesenchymal markers such as $\mathrm{N}$-cadherin, vimentin and

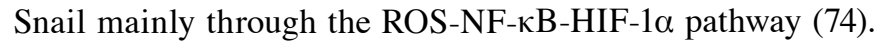
Another research on human skin squamous cell carcinoma and melanoma described that autophagy deficiency facilitated EMT by stabilizing the pivotal mesenchymal marker TWIST1 (75). Autophagy stimulation downregulated two key regulators Slug and Snail in glioblastoma cells while inhibition of ATG5 and ATG7 led to overexpression of Slug and Snail (76). Studies on breast and colon cancers described that the death effector domain-containing DNA-binding protein (DEDD) negatively regulated EMT by activating autophagy and then inducing the autophagy-mediated lysosomal degradation of Snail and
Twist (77). Considering its special role in supporting cell viability during cancer progression and migration, autophagy also has a positive effect on EMT. Li and his colleagues (78) found that the inhibition of autophagy by silencing ATG3 or ATG7 also suppressed EMT and TGF- $\beta / \mathrm{Smad} 3$ signaling in hepatocellular carcinoma cells HepG2 and BEL 7402. While starvation-induced autophagy can promote EMT through the TGF- $\beta$ /Smad3 signaling-dependent manner.

The correlation between autophagy and EMT is largely based on the close relationship between cytoskeleton and mitochondria and their pivotal function in modulating the two processes. Cytoskeleton structures are essential to facilitate cell movement and cytoskeleton remodeling is indispensable to accomplish the process of EMT $(79,80)$. While mitochondria are responsible for ATP production and play fundamental roles in maintaining cellular metabolic homeostasis (81). Mitochondria are dynamic organelles that experience fusion and fission continuously (82). Fissile mitochondria are degraded through autophagy to be reused as source of energy and thus completed the recycling of metabolites $(14,81)$. Mitochondria are reticular organelles characterized as high plasticity to move across the cells through the cytoskeleton (81). Amassing of mitochondria below the cell membrane is essential to provide an abundance of ATP to upgrade the formation of lamellipodia and filopodia, and then assuring the cellular motility during $\operatorname{EMT}(83,84)$.

Thus, the close relationship between mitochondria and cytoskeleton is correlated with both EMT and autophagy. During cancer progression, mitochondrial dynamics provide ATP for cytoskeleton remodeling to promote EMT while autophagy regulates mitochondrial dynamics by eliminating the damaged mitochondria. The relationship between autophagy and EMT in cancer is presented in Fig. 3. 


\section{Perspectives}

Inactivation of tumor suppressor genes and activation of oncogenes may collaborate to induce cell malignant transformation. RAS and p53 have been found most frequently mutated in majority of human cancers. Early studies revealed that the activated H-RAS ${ }^{\mathrm{V} 12}$ cooperates with mutant $\mathrm{p} 53$ to induce tumor progression (85-87). Recent studies report that mutant p53 cooperates with activated RAS to stimulate highly invasive and metastatic tumors with poor prognosis (88-92). Since RAS and 553 pathways function as pivotal regulators in both cancer cells and tumor microenvironment (93), the associated genes including HIF-1 $\alpha$, HDAC, EHF and VGLL and their functions may be thoroughly examined in autophagy and EMT. Retention of wt p53 can facilitate the sensitivity to chemotherapy in some tumor types and inhibition of the RAS downstream signaling factor AKT also represses survival, invasiveness and drug resistance of cancer cells (94-96). A variety of molecules and existing therapeutic agents targeting the RAS and p53 pathways are currently in clinical trials $(97,98)$. Thus, identification of novel molecules or signaling cascades involved with $\mathrm{p} 53$ or RAS mutations may greatly contribute to precision medicine toward cancer treatment and prevention.

\section{Acknowledgements}

The present review was supported by grants from the National Natural Science Foundation of China (nos. 81572553 and 81372797 to G.Y.).

\section{References}

1. Stępiński D: Nucleolus-derived mediators in oncogenic stress response and activation of p53-dependent pathways. Histochem Cell Biol 146: 119-139, 2016.

2. Merino D and Malkin D: p53 and hereditary cancer. Subcell Biochem 85: 1-16, 2014.

3. Muller PA and Vousden KH: p53 mutations in cancer. Nat Cell Biol 15: 2-8, 2013.

4. Freed-Pastor WA and Prives C: Mutant p53: One name, many proteins. Genes Dev 26: 1268-1286, 2012.

5. Silva JL, De Moura Gallo CV, Costa DC and Rangel LP: Prionlike aggregation of mutant $\mathrm{p} 53$ in cancer. Trends Biochem Sci 39: 260-267, 2014.

6. Fang B: RAS signaling and anti-RAS therapy: Lessons learned from genetically engineered mouse models, human cancer cells, and patient-related studies. Acta Biochim Biophys Sin (Shanghai) 48: 27-38, 2016.

7. Kimmelman AC: Metabolic dependencies in RAS-driven cancers. Clin Cancer Res 21: 1828-1834, 2015.

8. Stites EC and Ravichandran KS: A systems perspective of ras signaling in cancer. Clin Cancer Res 15 (5): 1510-1513, 2009.

9. Vandal G, Geiling B and Dankort D: Ras effector mutant expression suggest a negative regulator inhibits lung tumor formation. PLoS One 9: e84745, 2014.

10. Xia M and Land H: Tumor suppressor p53 restricts Ras stimulation of RhoA and cancer cell motility. Nat Struct Mol Biol 14: 215-223, 2007.

11. Meylan E, Dooley AL, Feldser DM, Shen L, Turk E, Ouyang C and Jacks T: Requirement for NF-kappaB signalling in a mouse model of lung adenocarcinoma. Nature 462: 104-107, 2009.

12. Boiko AD, Porteous S, Razorenova OV, Krivokrysenko VI, Williams BR and Gudkov AV: A systematic search for downstream mediators of tumor suppressor function of $\mathrm{p} 53$ reveals a major role of BTG2 in suppression of Ras-induced transformation. Genes Dev 20: 236-252, 2006.

13. Song $\mathrm{H}$, Hollstein $\mathrm{M}$ and $\mathrm{Xu} \mathrm{Y}$ : p53 gain-of-function cancer mutants induce genetic instability by inactivating ATM. Nat Cell Biol 9: 573-580, 2007.
14. Lorin S, Hamaï A, Mehrpour M and Codogno P: Autophagy regulation and its role in cancer. Semin Cancer Biol 23: 361-379, 2013.

15. Mizushima N, Levine B, Cuervo AM and Klionsky DJ: Autophagy fights disease through cellular self-digestion. Nature 451: 1069-1075, 2008.

16. Kumar A, Singh UK and Chaudhary A: Targeting autophagy to overcome drug resistance in cancer therapy. Future Med Chem 7: 1535-1542, 2015.

17. White E and DiPaola RS: The double-edged sword of autophagy modulation in cancer. Clin Cancer Res 15: 5308-53016, 2009.

18. Tang J, Di J, Cao H, Bai J and Zheng J: p53-mediated autophagic regulation: A prospective strategy for cancer therapy. Cancer Lett 363: 101-107, 2015.

19. Tasdemir E, Maiuri MC, Galluzzi L, Vitale I, DjavaheriMergny M, D'Amelio M, Criollo A, Morselli E, Zhu C, Harper $\mathrm{F}$, et al: Regulation of autophagy by cytoplasmic p53. Nat Cell Biol 10: 676-687, 2008.

20. Schmukler E, Kloog Y and Pinkas-Kramarski R: Ras and autophagy in cancer development and therapy. Oncotarget 5: 577-586, 2014.

21. Lock R, Kenific CM, Leidal AM, Salas E and Debnath J: Autophagy-dependent production of secreted factors facilitates oncogenic RAS-driven invasion. Cancer Discov 4: 466-479, 2014.

22. White E: Deconvoluting the context-dependent role for autophagy in cancer. Nat Rev Cancer 12: 401-410, 2012.

23. Budanov AV: Stress-responsive sestrins link p53 with redox regulation and mammalian target of rapamycin signaling. Antioxid Redox Signal 15: 1679-1690, 2011.

24. Huang $J$ and Manning BD: The TSC1-TSC2 complex: A molecular switchboard controlling cell growth. Biochem J 412: 179-190, 2008.

25. Gwinn DM, Shackelford DB, Egan DF, Mihaylova MM, Mery A, Vasquez DS, Turk BE and Shaw RJ: AMPK phosphorylation of raptor mediates a metabolic checkpoint. Mol Cell 30: 214-226, 2008.

26. Kong B, Wu W, Cheng T, Schlitter AM, Qian C, Bruns P, Jian Z, Jäger C, Regel I, Raulefs S, et al: A subset of metastatic pancreatic ductal adenocarcinomas depends quantitatively on oncogenic Kras/Mek/Erk-induced hyperactive mTOR signalling. Gut 65: 647-657, 2016.

27. Kim J, Kundu M, Viollet B and Guan KL: AMPK and mTOR regulate autophagy through direct phosphorylation of Ulk1. Nat Cell Biol 13: 132-141, 2011.

29. Mizushima N, Yoshimori T and Ohsumi Y: The role of Atg proteins in autophagosome formation. Annu Rev Cell Dev Biol 27: 107-132, 2011.

30. Eby KG, Rosenbluth JM, Mays DJ, Marshall CB, Barton CE, Sinha S, Johnson KN, Tang L and Pietenpol JA: ISG20L1 is a p53 family target gene that modulates genotoxic stress-induced autophagy. Mol Cancer 9: 95, 2010.

31. Kenzelmann Broz D, Spano Mello S, Bieging KT, Jiang D, Dusek RL, Brady CA, Sidow A and Attardi LD: Global genomic profiling reveals an extensive p53-regulated autophagy program contributing to key p53 responses. Genes Dev 27: 1016-1031, 2013.

32. Gao W, Shen Z, Shang L and Wang X: Upregulation of human autophagy-initiation kinase ULK1 by tumor suppressor p53 contributes to DNA-damage-induced cell death. Cell Death Differ 18: 1598-1607, 2011.

33. Guo JY, Karsli-Uzunbas G, Mathew R, Aisner SC, Kamphorst JJ, Strohecker AM, Chen G, Price S, Lu W, Teng X, et al: Autophagy suppresses progression of K-ras-induced lung tumors to oncocytomas and maintains lipid homeostasis. Genes Dev 27: 1447-1461, 2013.

34. Kim MJ, Woo SJ, Yoon CH, Lee JS, An S, Choi YH, Hwang SG, Yoon $\mathrm{G}$ and Lee SJ: Involvement of autophagy in oncogenic K-Ras-induced malignant cell transformation. J Biol Chem 286: 12924-12932, 2011.

36. Kinsey C, Balakrishnan V, O'Dell MR, Huang JL, Newman L, Whitney-Miller CL, Hezel AF and Land H: Plac8 links oncogenic mutations to regulation of autophagy and is critical to pancreatic cancer progression. Cell Rep 7: 1143-1155, 2014.

37. Desai S, Liu Z, Yao J, Patel N, Chen J, Wu Y, Ahn EE, Fodstad O and Tan M: Heat shock factor 1 (HSF1) controls chemoresistance and autophagy through transcriptional regulation of autophagyrelated protein 7 (ATG7). J Biol Chem 288: 9165-9176, 2013. 
38. Vydra N, Toma A and Widlak W: Pleiotropic role of HSF1 in neoplastic transformation. Curr Cancer Drug Targets 14: 144-155, 2014.

39. Dai C, Santagata S, Tang Z, Shi J, Cao J, Kwon H, Bronson RT, Whitesell L and Lindquist S: Loss of tumor suppressor NF1 activates HSF1 to promote carcinogenesis. J Clin Invest 122 3742-3754, 2012.

40. Li Q and Martinez JD: P53 is transported into the nucleus via an Hsf1-dependent nuclear localization mechanism. Mol Carcinog 50: 143-152, 2011.

41. Hu YL, Jahangiri A, De Lay M and Aghi MK: Hypoxia-induced tumor cell autophagy mediates resistance to anti-angiogenic therapy. Autophagy 8: 979-981, 2012.

43. Chen C, Pore N, Behrooz A, Ismail-Beigi F and Maity A: Regulation of glut1 mRNA by hypoxia-inducible factor-1. Interaction between $\mathrm{H}$-ras and hypoxia. J Biol Chem 276 : 9519-9525, 2001.

44. Nieminen AL, Qanungo S, Schneider EA, Jiang BH and Agani FH: Mdm2 and HIF-1alpha interaction in tumor cells during hypoxia. J Cell Physiol 204: 364-369, 2005.

45. Robertson ED, Semenchenko K and Wasylyk B: Crosstalk between Mdm2, p53 and HIF1- $\alpha$ : Distinct responses to oxygen stress and implications for tumour hypoxia. Subcell Biochem 85 : 199-214, 2014

46. Hay ED: An overview of epithelio-mesenchymal transformation Acta Anat (Basel) 154: 8-20, 1995.

47. Lamouille S, Xu J and Derynck R: Molecular mechanisms of epithelial-mesenchymal transition. Nat Rev Mol Cell Biol 15 178-196, 2014

48. Puisieux A, Brabletz T and Caramel J: Oncogenic roles of EMT-inducing transcription factors. Nat Cell Biol 16: 488-494, 2014.

49. Fischer KR, Durrans A, Lee S, Sheng J, Li F, Wong ST, Choi H, El Rayes T, Ryu S, Troeger J, et al: Epithelial-to-mesenchyma transition is not required for lung metastasis but contributes to chemoresistance. Nature 527: 472-476, 2015.

50. Zheng X, Carstens JL, Kim J, Scheible M, Kaye J, Sugimoto H Wu CC, LeBleu VS and Kalluri R: Epithelial-to-mesenchymal transition is dispensable for metastasis but induces chemoresistance in pancreatic cancer. Nature 527: 525-530, 2015.

51. Wang Y, Ngo VN, Marani M, Yang Y, Wright G, Staudt LM and Downward J: Critical role for transcriptional repressor Snail2 in transformation by oncogenic RAS in colorectal carcinoma cells. Oncogene 29: 4658-4670, 2010.

52. Gonzalez DM and Medici D: Signaling mechanisms of the epithelial-mesenchymal transition. Sci Signal 7: re8, 2014.

53. Zhang J, Lei Y, Gao X, Liang Q, Li L, Feng J, Hou P, Han L, Zhang Y, Huang B, et al: p53 Attenuates the oncogenic Ras-induced epithelial-mesenchymal transition in human mammary epithelial cells. Biochem Biophys Res Commun 434 606-613, 2013

54. Wang Z, Wade P, Mandell KJ, Akyildiz A, Parkos CA, Mrsny RJ and Nusrat A: Raf 1 represses expression of the tight junction protein occludin via activation of the zinc-finger transcription factor slug. Oncogene 26: 1222-1230, 2007.

55. Saegusa M,Hashimura M, Kuwata T and Okayasu I: Requirement of the Akt/beta-catenin pathway for uterine carcinosarcoma genesis, modulating E-cadherin expression through the transactivation of slug. Am J Pathol 174: 2107-2115, 2009.

56. Wang SP, Wang WL, Chang YL, Wu CT, Chao YC, Kao SH, Yuan A, Lin CW, Yang SC, Chan WK, et al: p53 controls cancer cell invasion by inducing the MDM2-mediated degradation of Slug. Nat Cell Biol 11: 694-704, 2009.

57. Wang Y, Bu F, Royer C, Serres S, Larkin JR, Soto MS, Sibson NR, Salter V, Fritzsche F, Turnquist C, et al: ASPP2 controls epithelial plasticity and inhibits metastasis through $\beta$-catenin-dependent regulation of ZEB1. Nat Cell Biol 16: 1092-1104, 2014.

58. Kim T, Veronese A, Pichiorri F, Lee TJ, Jeon YJ, Volinia S, Pineau P, Marchio A, Palatini J, Suh SS, et al: p53 regulates epithelial-mesenchymal transition through microRNAs targeting ZEB1 and ZEB2. J Exp Med 208: 875-883, 2011

59. Chang CJ, Chao CH, Xia W, Yang JY, Xiong Y, Li CW, Yu WH Rehman SK, Hsu JL, Lee HH, et al: p53 regulates epithelialmesenchymal transition and stem cell properties through modulating miRNAs. Nat Cell Biol 13: 317-323, 2011

60. Ansieau S, Bastid J, Doreau A, Morel AP, Bouchet BP, Thomas C, Fauvet F, Puisieux I, Doglioni C, Piccinin S, et al: Induction of EMT by twist proteins as a collateral effect of tumor-promoting inactivation of premature senescence. Cancer Cell 14: 79-89, 2008 .
61. Jiang Y, Xie X, Li Z, Wang Z, Zhang Y, Ling ZQ, Pan Y, Wang Z and Chen Y: Functional cooperation of RKTG with p53 in tumorigenesis and epithelial-mesenchymal transition. Cancer Res 71: 2959-2968, 2011.

62. Gibbons DL, Lin W, Creighton CJ, Rizvi ZH, Gregory PA, Goodall GJ, Thilaganathan N, Du L, Zhang Y, Pertsemlidis A, et al: Contextual extracellular cues promote tumor cell EMT and metastasis by regulating miR-200 family expression. Genes Dev 23: 2140-2151, 2009.

63. Roger L, Jullien L, Gire V and Roux P: Gain of oncogenic function of p53 mutants regulates E-cadherin expression uncoupled from cell invasion in colon cancer cells. J Cell Sci 123: 1295-1305, 2010.

64. Ohashi S, Natsuizaka M, Wong GS, Michaylira CZ, Grugan KD, Stairs DB, Kalabis J, Vega ME, Kalman RA, Nakagawa M, et al: Epidermal growth factor receptor and mutant p53 expand an esophageal cellular subpopulation capable of epithelial-tomesenchymal transition through ZEB transcription factors. Cancer Res 70: 4174-4184, 2010.

65. Jiang Z, Deng T, Jones R, Li H, Herschkowitz JI, Liu JC, Weigman VJ, Tsao MS, Lane TF, Perou CM, et al: Rb deletion in mouse mammary progenitors induces luminal-B or basal-like/ EMT tumor subtypes depending on p53 status. J Clin Invest 120: 3296-3309, 2010

66. Okada T, Sinha S, Esposito I, Schiavon G, López-Lago MA, Su W, Pratilas CA, Abele C, Hernandez JM, Ohara M, et al: The Rho GTPase Rnd1 suppresses mammary tumorigenesis and EMT by restraining Ras-MAPK signalling. Nat Cell Biol 17: 81-94, 2015.

67. Gao T, Li JZ, Lu Y, Zhang CY, Li Q, Mao J and Li LH: The mechanism between epithelial mesenchymal transition in breast cancer and hypoxia microenvironment. Biomed Pharmacother 80: 393-405, 2016.

68. Imai T, Horiuchi A, Wang C, Oka K, Ohira S, Nikaido T and Konishi I: Hypoxia attenuates the expression of E-cadherin via up-regulation of SNAIL in ovarian carcinoma cells. Am J Pathol 163: 1437-1447, 2003.

69. Zhang L, Huang G, Li X, Zhang Y, Jiang Y, Shen J, Liu J, Wang Q, Zhu J, Feng X, et al: Hypoxia induces epithelial-mesenchymal transition via activation of SNAI1 by hypoxia-inducible factor-1a in hepatocellular carcinoma. BMC Cancer 13: 108, 2013.

70. Cui Y, Li YY, Li J, Zhang HY, Wang F, Bai X and Li SS: STAT3 regulates hypoxia-induced epithelial mesenchymal transition in oesophageal squamous cell cancer. Oncol Rep 36: 108-116, 2016.

71. Tsai YP and Wu KJ: Hypoxia-regulated target genes implicated in tumor metastasis. J Biomed Sci 19: 102, 2012.

72. Tsai JH and Yang J: Epithelial-mesenchymal plasticity in carcinoma metastasis. Genes Dev 27: 2192-2206, 2013.

73. Gugnoni M, Sancisi V, Manzotti G, Gandolfi G and Ciarrocchi A: Autophagy and epithelial-mesenchymal transition: An intricate interplay in cancer. Cell Death Dis 7: e2520, 2016.

74. Qin W, Li C, Zheng W, Guo Q, Zhang Y, Kang M, Zhang B, Yang B, Li B, Yang H, et al: Inhibition of autophagy promotes metastasis and glycolysis by inducing ROS in gastric cancer cells. Oncotarget 6: 39839-39854, 2015 .

75. Qiang L and He YY: Autophagy deficiency stabilizes TWIST1 to promote epithelial-mesenchymal transition. Autophagy 10: 1864-1865, 2014

76. Catalano M, D'Alessandro G, Lepore F, Corazzari M, Caldarola S, Valacca C, Faienza F, Esposito V, Limatola C, Cecconi F, et al: Autophagy induction impairs migration and invasion by reversing EMT in glioblastoma cells. Mol Oncol 9: 1612-1625, 2015.

77. Lv Q, Hua F and Hu ZW: DEDD, a novel tumor repressor, reverses epithelial-mesenchymal transition by activating selective autophagy. Autophagy 8: 1675-1676, 2012.

78. Li J, Yang B, Zhou Q, Wu Y, Shang D, Guo Y, Song Z, Zheng Q and Xiong J: Autophagy promotes hepatocellular carcinoma cell invasion through activation of epithelial-mesenchymal transition. Carcinogenesis 34: 1343-1351, 2013.

79. Wei SC and Yang J: Forcing through tumor metastasis: The interplay between tissue rigidity and epithelial-mesenchymal transition. Trends Cell Biol 26: 111-120, 2016.

80. Tojkander S, Gateva G and Lappalainen P: Actin stress fibers-assembly, dynamics and biological roles. J Cell Sci 125: 1855-1864, 2012

81. Ni HM, Williams JA and Ding WX: Mitochondrial dynamics and mitochondrial quality control. Redox Biol 4: 6-13, 2015.

82. Youle RJ and van der Bliek AM: Mitochondrial fission, fusion, and stress. Science 337: 1062-1065, 2012. 
83. Zhao J, Zhang J, Yu M, Xie Y, Huang Y, Wolff DW, Abel PW and Tu Y: Mitochondrial dynamics regulates migration and invasion of breast cancer cells. Oncogene 32: 4814-4824, 2013.

84. Ketschek A and Gallo G: Nerve growth factor induces axonal filopodia through localized microdomains of phosphoinositide 3-kinase activity that drive the formation of cytoskeletal precursors to filopodia. J Neurosci 30: 12185-12197, 2010.

85. Parada LF, Land H, Weinberg RA, Wolf D and Rotter V: Cooperation between gene encoding p53 tumour antigen and ras in cellular transformation. Nature 312: 649-651, 1984.

86. Jenkins JR, Rudge K and Currie GA: Cellular immortalization by a cDNA clone encoding the transformation-associated phosphoprotein p53. Nature 312: 651-654, 1984.

87. Eliyahu D, Raz A, Gruss P, Givol D and Oren M: Participation of p53 cellular tumour antigen in transformation of normal embryonic cells. Nature 312: 646-649, 1984.

88. DuPage M, Dooley AL and Jacks T: Conditional mouse lung cancer models using adenoviral or lentiviral delivery of Cre recombinase. Nat Protoc 4: 1064-1072, 2009.

89. Hingorani SR, Wang L, Multani AS, Combs C, Deramaudt TB, Hruban RH, Rustgi AK, Chang S and Tuveson DA: Trp53 $3^{R 172 H}$ and $\operatorname{Kras}^{G 12 D}$ cooperate to promote chromosomal instability and widely metastatic pancreatic ductal adenocarcinoma in mice. Cancer Cell 7: 469-483, 2005.

90. Tsumura H, Yoshida T, Saito H, Imanaka-Yoshida K and Suzuki N: Cooperation of oncogenic K-ras and p53 deficiency in pleomorphic rhabdomyosarcoma development in adult mice. Oncogene 25: 7673-7679, 2006.

91. Zheng S, El-Naggar AK, Kim ES, Kurie JM and Lozano G: A genetic mouse model for metastatic lung cancer with gender differences in survival. Oncogene 26: 6896-6904, 2007.
92. Muñoz DM, Tung T, Agnihotri S, Singh S, Guha A, Zadeh G and Hawkins C: Loss of p53 cooperates with K-ras activation to induce glioma formation in a region-independent manner. Glia 61: 1862-1872, 2013.

93. Solomon H, Brosh R, Buganim Y and Rotter V: Inactivation of the p53 tumor suppressor gene and activation of the Ras oncogene: Cooperative events in tumorigenesis. Discov Med 9: 448-454, 2010.

94. Jackson JG and Lozano G: The mutant p53 mouse as a preclinical model. Oncogene 32: 4325-4330, 2013

95. Bertheau P, Turpin E, Rickman DS, Espié M, de Reyniès A, Feugeas JP, Plassa LF, Soliman H, Varna M, de Roquancourt A, et al: Exquisite sensitivity of TP53 mutant and basal breast cancers to a dose-dense epirubicin-cyclophosphamide regimen. PLoS Med 4: e90, 2007.

96. Cassinelli G, Zuco V, Gatti L, Lanzi C, Zaffaroni N, Colombo D and Perego P: Targeting the Akt kinase to modulate survival, invasiveness and drug resistance of cancer cells. Curr Med Chem 20: 1923-1945, 2013.

97. Gurpinar E and Vousden KH: Hitting cancers' weak spots: Vulnerabilities imposed by p53 mutation. Trends Cell Biol 25: 486-495, 2015.

98. Bournet B, Buscail C, Muscari F, Cordelier P and Buscail L: Targeting KRAS for diagnosis, prognosis, and treatment of pancreatic cancer: Hopes and realities. Eur J Cancer 54: 75-83, 2016. 